\title{
Abnormal softening of Ti-metallic glasses during nanosecond laser shock peening
}

\author{
Yansen $\mathrm{Li}^{\text {a,c }}{ }^{\text {, Kun Zhang }}{ }^{\text {a,c, *** }}$, Yang Wang ${ }^{\text {a,c }}$, Weiqi Tang ${ }^{\text {a,c }}$, Yating Zhang ${ }^{\text {a,c }}$, \\ Bingchen Wei ${ }^{\text {a,b, c, }}$, Zheng $\mathrm{Hu}^{\mathrm{d}}$ \\ ${ }^{a}$ CAS Key Laboratory of Microgravity (National Microgravity Laboratory), Institute of Mechanics, Chinese Academy of Sciences, Beijing, 100190, China \\ ${ }^{\mathrm{b}}$ Center of Materials Science and Optoelectronics Engineering, University of Chinese Academy of Sciences, Beijing, 100049, China \\ ${ }^{\mathrm{c}}$ School of Engineering Science, University of Chinese Academy of Sciences, Beijing, 100049, China \\ ${ }^{\mathrm{d}}$ Science and Technology on Vehicle Transmission Laboratory, China North Vehicle Research Institute, Beijing, 100072, China
}

\section{A R T I C L E I N F O}

\section{Keywords:}

Laser shock wave

Metallic glass

Grid nanoindentation

\begin{abstract}
A B S T R A C T
Nanosecond laser shock peening (NLSP) has been proved to be an effective way to enhance the mechanical properties of metallic components through modifying the surface microstructures. However, the micromechanism of structure and property fluctuations induced by shock wave propagation is still limited due to the intrinsic defects in crystalline materials. Here, NLSP treatment has been performed into the Ti-metallic glasses. Owing to the absence of dislocations, grain boundary and phase segregation, the structural signals caused by the shock wave could come into sight. According to grid nanoindentation results, the shock-affected region can be divided into three sections: (I) the rapid softening region, (II) the recovery region and (III) the matrix region. The fronted first pop-in event and the reduced hardness found in the subsurface region provide clear evidence of the release wave-induced structural rearrangement. The abnormal change of the average volume of the shear transformation zone (STZ) could be explained by a two-step transformation mechanism, due to the fluctuations of the "weak spot" induced by the release stress wave. By means of these results, this work provides an opportunity to shed light on the common laser-solid interaction.
\end{abstract}

\section{Introduction}

As an attractive surface treatment technique, Nanosecond laser shock peening (NLSP) is usually used to modify metallic component surface and enhance their mechanical properties [1-3]. This technique relies on a powerful compressive shock wave generated by a high energy density, very short duration laser pulse, which propagates from the surface deep into the target material to induce a strong compressive residual stress that strengthens it [4]. NLSP has obvious advantages, such as no contact and heat-affected zone, exceptional controllability and impact [5]. Earlier research has shown that NLSP can enhance the fatigue and wear resistance performance in the presence of near-surface work-hardened layers in conventional metal materials [6-8]. The increase of strength and hardness was attributed to the slip, twinning and network of tangled dislocations [9]. NLSP can also be recommended as an effective method for reducing brittleness in metallic glasses (MGs). The effect of NLSP on the mechanical properties has been studied widely, which is beneficial for plasticity improvement in MGs due to the appearance of the extra-deep residual stress region, structural rejuvenation and abundant embryonic shear bands [10-13]. In addition, the combined effect of high hydrostatic and shear stresses was found to be able to induce a crystalline-to-amorphous transformation when the shock pressure was above a critical threshold value [14,15].

Although the response of materials to NLSP has been studied for decades, our understanding of the micro-scale response of metals to rapid loading remains limited. The residual stress or microhardness usually reaches its maximum value nearby the top surface and slowly decreases along the laser shocked direction $[16,17]$. However, a long laser pulse duration and high power density may give rise to the loss of the compressive stress in the laser spot center in some metal systems [18,

\footnotetext{
* Corresponding author. CAS Key Laboratory of Microgravity (National Microgravity Laboratory), Institute of Mechanics, Chinese Academy of Sciences, Beijing, 100190, China.

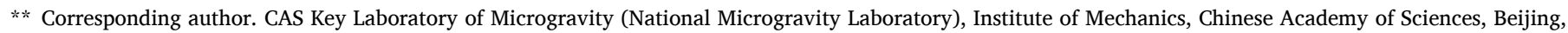
100190, China.
}

E-mail addresses: zhangkun@imech.ac.cn (K. Zhang), weibc@imech.ac.cn (B. Wei). 
19]. The extremes of pressure and temperature produced by shock compression may significantly hinder the interaction between the microstructural change and shock wave propagation. On the other hand, intrinsic defects in metals, such as dislocations, grain boundary and phase separations, may cause the impedance mismatch, which in turn increases complexity for obtaining the evolution of atomic rearrangement during the propagation of the shock wave [20,21]. A full description of shock-induced elastic-plastic deformation is still required in some simple systems with simple method. Fortunately, MGs can be viewed as suitable candidates for investigating the underlying interaction between the shock wave and solid, due to the absence of dislocations, grain boundary and phase segregation. In addition, numerous experimental studies and computational simulations have revealed the existence of micro spatial heterogeneity in MGs, which could serve as a feasible structural indicator to evaluate their mechanical properties and deformation behavior [22-24]. These shock wave-induced microstructural fluctuations may also provide a new approach to gain a better understanding of the common laser-solid interactions.

The nanoindentation test is commonly used today to explore the mechanical properties of MGs, where the depth-sensing method produces a representative load-displacement curve from the quantitative value of the properties [25-27]. Other traditional methods can be replaced by a novel grid nanoindentation test, which not only can be used as a two-dimensional (2D) tool to determine the local elastic and plastic properties of the shock-affected region, but also gain insight into the understanding of the structural rearrangement and fluctuations of MGs at a particular length scale.

In this study, the local mechanical properties of NLSP-treated Timetallic glasses (Ti-MGs) were studied by grid nanoindentation method. A large compressive stress loss region was observed around the laser spot center. It can be attributed to the dynamical rebound of the stress wave in the boundary, which corresponds to an edge effect arising from the impossibility of a discontinuity existing between the inner and outer sides of the impact region [28]. The structural rearrangements and local plastic deformation at the micro-scale are also investigated.

\section{Experimental details}

\subsection{Preparation of the samples}

A master alloy of $\mathrm{Ti}_{34.3} \mathrm{Zr}_{31.5} \mathrm{Ni}_{5.5} \mathrm{Cu}_{5} \mathrm{Be}_{23.7}$ (at.\%) was prepared by arc melting high pure metals $(99.99 \%)$ in a Ti-gettered argon atmosphere. The ingots were remelted more than 5 times to ensure compositional homogeneity, followed by suction casting into a copper mold. Eventually, a 5-mm diameter rod was obtained.

\subsection{Experimental procedures}

Two specimens $\left(20 \times 4 \times 3 \mathrm{~mm}^{3}\right)$ cut off from the cylindrical samples were clamped with the adjacent surfaces polished. The gap between them was less than $5 \mu \mathrm{m}$. Then, the specimens were attached to the backplate with glue. A Q-switched Nd: YAG pulse laser with $1064 \mathrm{~nm}$ wavelength was used to treat the samples. The diameter of the focused laser spot was about $2 \mathrm{~mm}$, resulting in the power density of $3.9 \mathrm{GW} /$ $\mathrm{cm}^{2}$. During the NLSP process, the ablative coating layer material covered on the target was aluminum foil, the purified water was chosen as the confining medium, as depicted in Fig. 1(a).

After NLSP treatment, the nanoindentation tests were conducted at room temperature using a Nano Indenter G200 with a Berkovich diamond indenter. The indentations were performed in load-controlled mode with the loading rate kept constant at $0.05 \mathrm{mN} / \mathrm{s}$. The maximum loading was fixed at $20 \mathrm{mN}$. For the sake of removing the thermal effect, the thermal drift below $0.05 \mathrm{~nm} / \mathrm{s}$ was maintained. The grid indentation was performed by using an indentation matrix $(8 \times 10$ indents), which covered about half of the impact region, as illustrated in Fig. 1(b).

\section{Results}

When the pressure wave penetrates the substrate surface and propagates as a shock wave, irreversible plastic deformations can be created deep into the shock affected region, which, in turn, can impact the mechanical properties and local structural rearrangement in Ti-MGs. The 2D maps of the local hardness, local residual plastic depth, local elastic modulus, and local viscosity in half of the shock-affected region along the laser beam direction, which were prepared by using data from the ultra-fast grid indentation experiments with a space of $100 \mu \mathrm{m}$, are shown in Fig. 2(a)-(d), respectively. The map in Fig. 2(a) reveals the presence of an obvious work-hardened layer on the surface resulting from the compressive effect of the shock wave. Different from conventional metals, the Ti-MGs in the hardness map display a distinct softening behavior in the sub-surface when the peened layer depth is increased, and then the value is restored at a deeper peened layer depth. The shock-affected region was approximately divided, according to the value of the local hardness, into three sections, namely the rapid softening region in subsurface (I), the recovery region in the middle (II) and the matrix region (III). The average value of the hardness in these regions was about 4.75, 6.17 and $7.17 \mathrm{GPa}$, respectively. Notable, the boundary of these regions exhibits an oval shape, which may be closely associated with the path of the shock wave propagation. Similarly, the local plastic depth also displayed a trend corresponding to the hardness

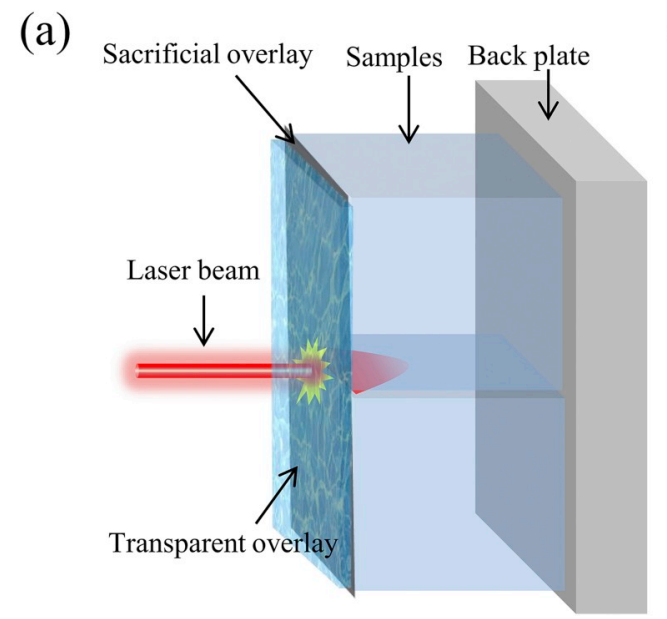

(b)

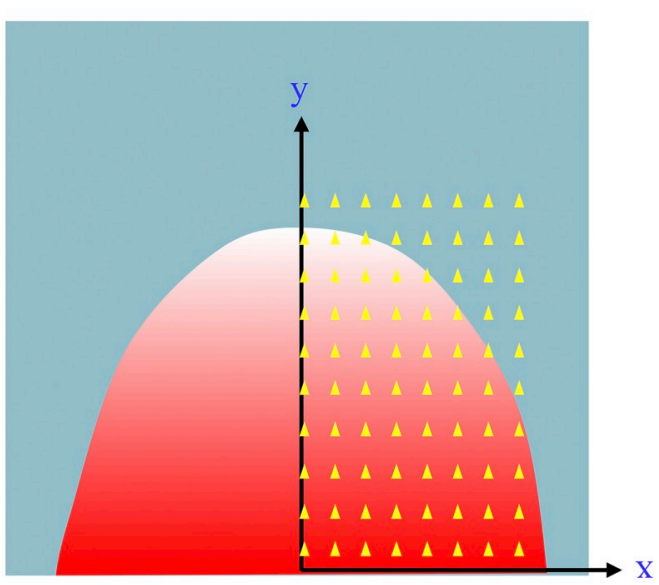

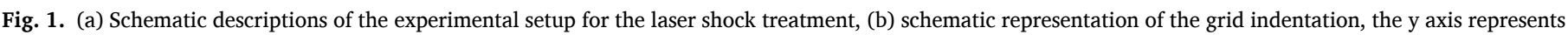
the incident laser beam direction. 

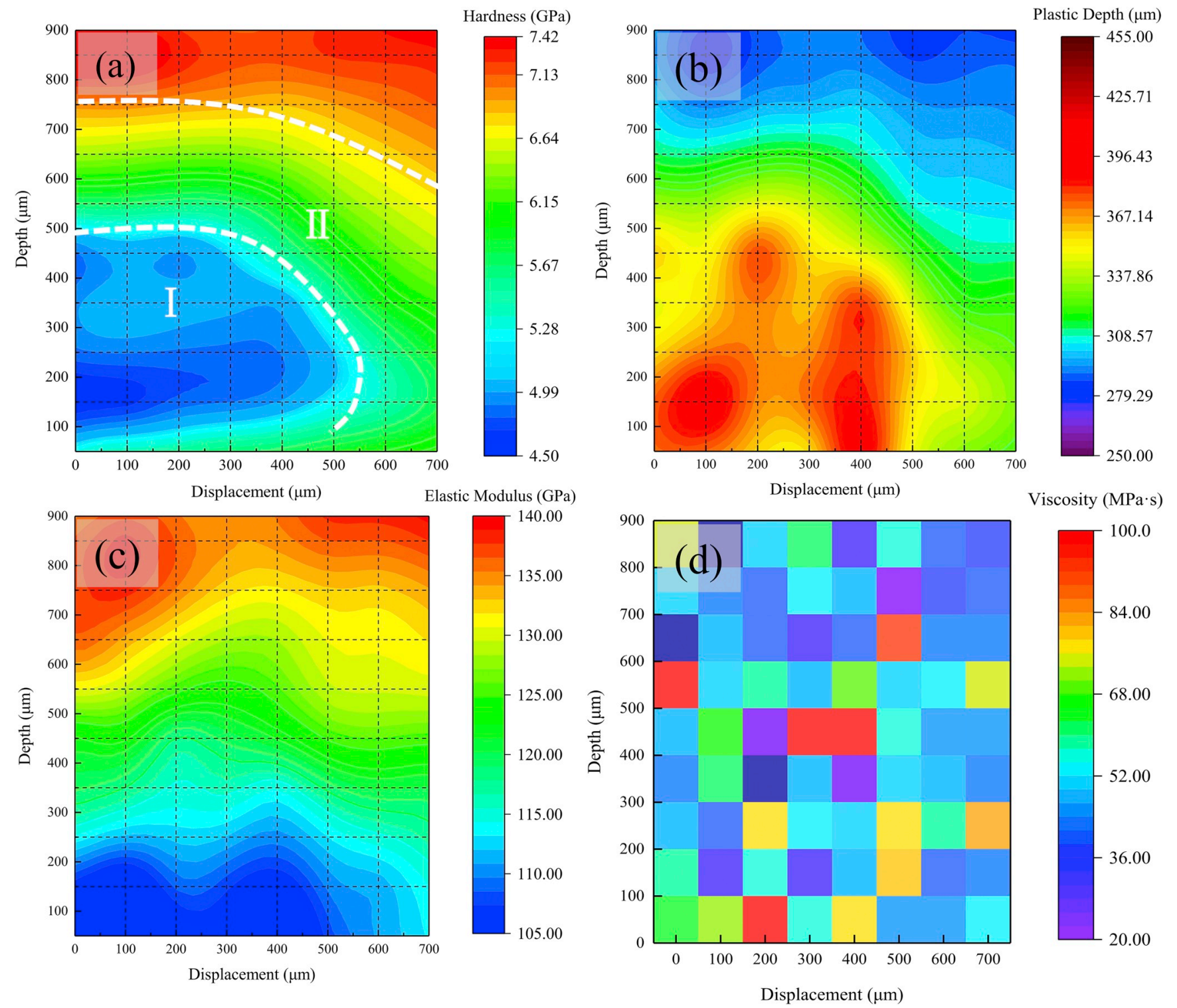

Fig. 2. 2D maps of (a) local hardness, (b) residual plastic depth, (c) elastic modulus and (d) viscosity.

in the map, as shown in Fig. 2(b). However, there is little consistency among the local elastic modulus, viscosity and hardness.

The indentation hardness and elastic modulus are critical parameters for evaluating the elastic-plastic deformation and plastic flow of the structural materials. Additionally, the relationship between them is of enormous interest to material research. The indentation hardness is conventionally defined as the average pressure under the indenter which resists to the permanent indentation. The elastic modulus is determined by the bonding forces between the constituent atoms, which depend on the interatomic distance. From the statistical trend, indentation hardness is viewed as a function of elastic modulus in crystalline structures. The ratio of hardness to elastic modulus is a key constraint, which reflects the ability for energy dissipation during plastic deformation [29]. For MGs with homogenous structure relative to their counterpart polycrystal, the ratio of hardness to elastic modulus follows the empirical relationship of $\mathrm{H} / \mathrm{E} \approx 0.05$ [30]. However, this is not the actual case in NLSP-treated MG systems. The data in Fig. 3(a) reveals that a good linear fit was achieved in the matrix region, where there was a high correlation with the empirical linear relationship, with a ratio of 0.055 . In the subsurface region, the ratio was about 0.064 , which is slightly larger than that in matrix region, while in the middle region, the ratio was close to 0.03 . The unique ratio of hardness to elastic modulus may serve as important evidence to understand the physical mechanisms underlying the laser-solid interaction, as will be discussed below.

The plastic deformation of MGs is essentially different from that in crystalline solids, which is usually caused by intense shearing in narrow shear bands [31]. The plastic flow is confined in the tiny space along the indenter tip. As a result, the shear band propagation is halted once the plastic strain is controlled by the cooperative atomic shear motion in the shear band. Accordingly, the initiation of plastic deformation in MGs during the nanoindentation process is frequently related to a displacement burst, or pop-in Ref. [32]. The first deviation from the elastic stage with the first pop-in event indicates a transformation to plastic deformation [33]. In order to systematically analyze the so-called "displacement burst" in the three above-mentioned regions, the representative P-h curves are supplied in Fig. 3(b), which exhibit the emblematic parabolic shape as well as obvious displacement bursts. Additionally, it is observed that the P-h curve in the matrix region shows the ideal stair-step-like serrations. In contrast, the pop-in events become weaker, and substantially transform into the ripple-shaped or even smooth parabolic curves in the middle or subsurface region. Essentially, the pop-in events represent the localized inhomogeneous plastic flow under 

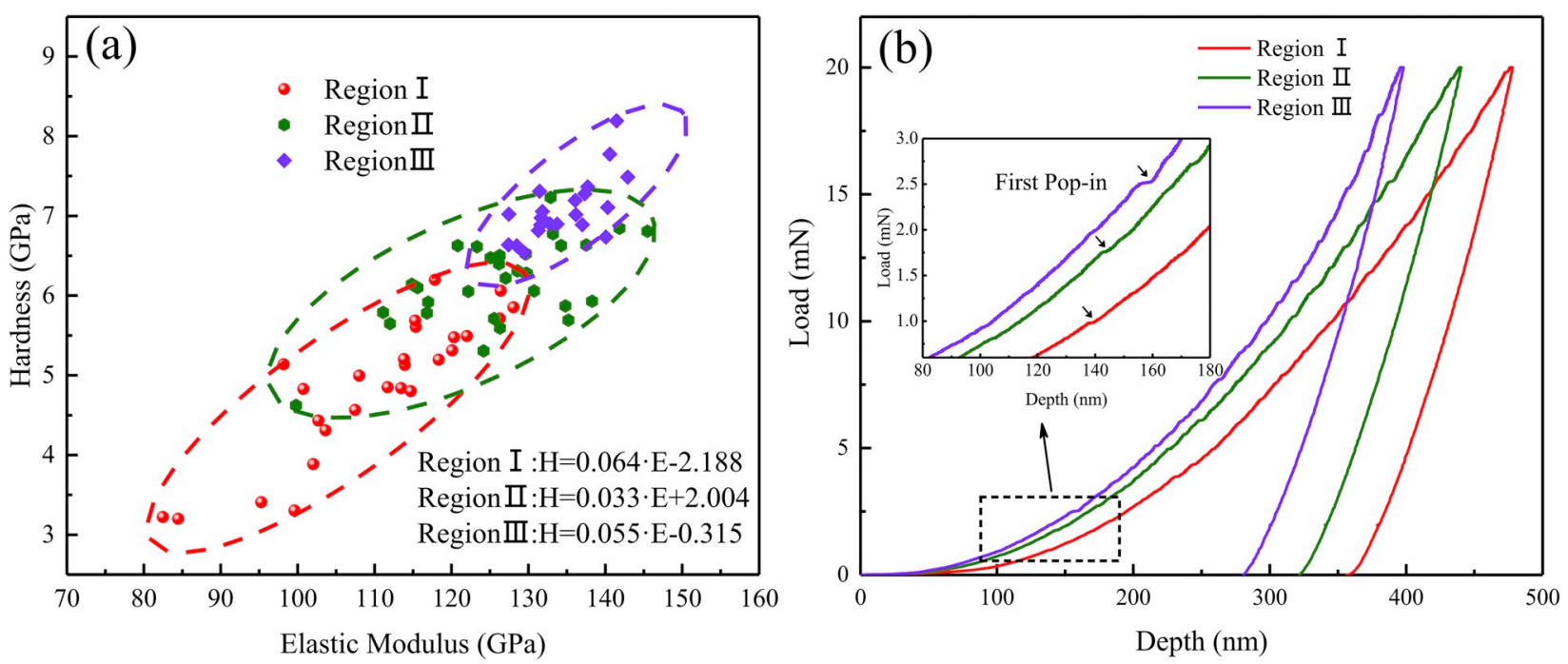

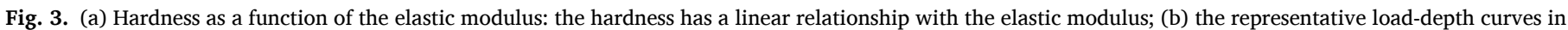

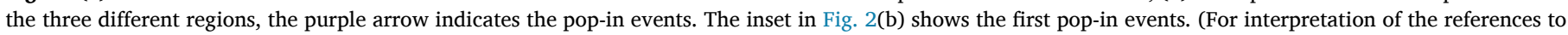
colour in this figure legend, the reader is referred to the Web version of this article.)

loading. With more pronounced serrations, the flow is more localized. In other words, weaker serrations mean better plastic deformation capacity. In our study, the loading rates for different impacted regions were kept constant. Thus, it can be expected that the capacity of plastic flow of MGs is changed by the laser shock wave. As shown in the inset of Fig. 3(b), the values of the applied load corresponding to the first pop-in events, $P_{c}$, were determined to be $2.47,1.76$, and $0.98 \mathrm{mN}$ for the matrix, middle and subsurface regions, respectively. This indicates that the first pop-in events occur gradually in advance in the subsurface region. The decrease of the intensity of the first pop-in event in the subsurface region may due to the reduced hardness and resistance to the shear event after NLSP treatment.

In thermal-mechanical coupled framework, the activation of the shear transformation underneath the indenter of MGs may occur in any stress level. The plastic deformation of MGs is controlled by a cooperative atomic rearrangement termed as STZ [34]. The STZ with dozens of atoms is preferentially rooted in the free volume or flow unit-riched regions. Thus, the structural rearrangement and fluctuations (the change of the content of free volume or flow unit) in different shock affected regions can effectively be identified from the activated state of the shear event. According to Hertzian elastic contact theory, the activation volume can be evaluated by means of the statistical analysis. The cumulative distribution of strength (Fig. 4) can be regarded as [35]:

$\mathrm{f}=1-\exp \left[-\frac{\mathrm{kT} \dot{\gamma}_{0}}{\mathrm{~V}^{*}(d \tau / \mathrm{dt})} \exp \left(-\frac{\Delta \mathrm{F}^{*}}{\mathrm{kT}}\right) \exp \left(\frac{\tau \mathrm{V}^{*}}{\mathrm{kT}}\right)\right]$

where $\mathrm{kT}$ denotes the thermal energy, $\Delta \mathrm{F}^{*}$ denotes Helmholtz activation energy, and $\mathrm{V}^{*}$ is the activation volume of the shear event. The $\mathrm{V}^{*}$ can be identified as the slope of the linear fit curves shown in inset of Fig. 4, and its value obtained by logarithmic conversion. The volume of STZ $(\Omega)$ can be expressed by Ref. [36]:

$\Omega=\frac{\tau_{\mathrm{C} 0}}{6 \mathrm{R}_{0} \mathrm{G}_{0} \gamma^{2} \xi\left(1-\tau_{\mathrm{CT}} / \tau_{\mathrm{C} 0}\right)^{1 / 2}} \mathrm{~V}^{*}$

where $\mathrm{G}_{0}$ denotes the shear modulus near absolute zero; $\tau_{\mathrm{CT}}$ and $\tau_{\mathrm{C} 0}$ represent the shear strengths at finite temperature and near absolute zero.

As shown in Fig. 4, a clear trend can be observed that the upper limit of $\tau_{\mathrm{m}}$ is shifted to smaller values from the matrix region to the subsurface region, indicating that the yielding occurs more easily after NLSP. The parameters for the three regions are shown in Table 1 . The volume

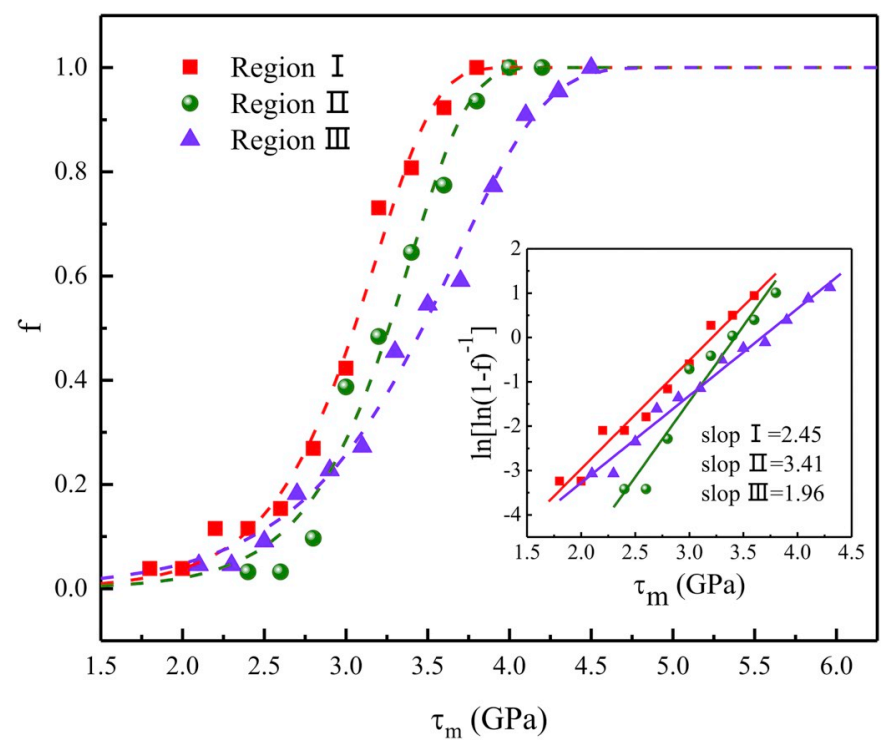

Fig. 4. The cumulative distribution of the shear strength associated with the first pop-in event. Inset: Linear fits to the $\ln [\ln (1-\mathrm{f})-1] \cdot \mathrm{kT}$ vs $\tau_{\mathrm{m}}$ curves, used to estimate the size of STZ in three different regions.

change of the STZ seems to be more complex in those three regions, which initially increased from the matrix region to the middle region, and then its value decreased from the middle region to the region near the surface. However, the value in the subsurface region is still higher than that in the matrix region. The structural rearrangement of MGs can

Table 1

The values of average hardness, activation volume of the shear event $\left(\mathrm{V}^{*}\right)$ and STZ volume $(\Omega)$ in different regions.

\begin{tabular}{llll}
\hline & $\begin{array}{l}\text { Average } \\
\text { hardness } \\
\mathrm{H}(\mathrm{GPa})\end{array}$ & $\begin{array}{l}\text { Activation volume of the shear event, } \\
\mathrm{V}^{*}\left(\mathrm{~nm}^{3}\right)\end{array}$ & $\begin{array}{l}\text { STZ } \\
\text { volume } \\
\Omega\left(\mathrm{nm}^{3}\right)\end{array}$ \\
\hline $\begin{array}{l}\text { region I } \\
\text { region II }\end{array}$ & 4.75 & 0.0101 & 0.214 \\
region & 7.18 & 0.0141 & 0.299 \\
$\quad$ III & & 0.0081 & 0.172 \\
\hline
\end{tabular}


be determined by the cooperative shear motion of STZ, which inherently depends on the activation volume. From the perspective of the "structural rejuvenation theory" [12,37], additional free volume or flow unit was preferentially activated in the subsurface region, the number and size of STZ should be the largest, which is contrary to the above-mentioned results. Thus, an unknown mechanism accounting for the change of $\Omega$ may exist, directing the interaction between solid and wave propagation.

\section{Discussion}

Several modelizations have been developed to estimate the induced shock wave formation and propagation based on a one-dimensional planar geometry, however, which were only valid for short laser pulse duration or moderate power density [38-40]. A good understanding of the residual stress or hardness distribution requires the accurate prediction of the stress wave propagation during NLSP process. As shown in Fig. 5(a), except for the main peak, a release wave was created in the subsurface region which tends to create tensile stresses [41]. Therefore, the structural rearrangements in the subsurface region can be significantly affected by the release waves owing to the change of stress state. It has been established that MGs are indeed microscopic heterogeneous with discretely distributed "weak spots" (the free volume or flow unit-riched regions) [42]. When the shock wave generated by laser penetrated MGs, it would promote the diffusive rearrangement of atoms and then lead to an increase of the volume fraction of "weak spots" and its length scale. However, the residual compressive stress may hinder the homogeneous nucleation of "weak spots" in the shocked affected regions. Due to the effect of the surface release wave, the atomic structures were rearranged at a particular length scale, in which the larger "weak spot" was decomposed into smaller one with concomitant increase in the number. The two-step transformation process is shown in Fig. 5(b). Thus, from a 2D perspective, MGs behave as a gradient fluctuation in both mechanical properties and microstructures in the shock affected region.

\section{Summary and conclusions}

In this study, the mechanical and structural fluctuations in Ti-MGs after NLSP treatment were characterized by the grid nanoindentation method. The shock-affected region was approximately divided, according to the dynamical rebound of the released stress wave, into three sections: the rapid softening region in the subsurface, the recovery region in the middle and the matrix region. The first pop-in event appearing early as well as the reduced hardness in the subsurface region may be attributed to the structural rearrangement and fluctuations induced by the release waves. The limit value $\tau_{\mathrm{m}}$ showed a similar trend to the nanoindentation hardness in the three regions, which was consistent with existence of a link between the elastic-plastic deformations in MGs. The average volume of STZ initially increased from the matrix region to the middle region, and then its value decreased from the middle region to the region near the surface. Additionally, a two-step transformation mechanism explained the abnormal change of STZ, due to the fluctuations of the 'weak spot' induced by the release stress wave.

\section{Declaration of competing interest}

We declare that we do not have any commercial or associative interest that represents a conflict of interest in connection with the work

(a)

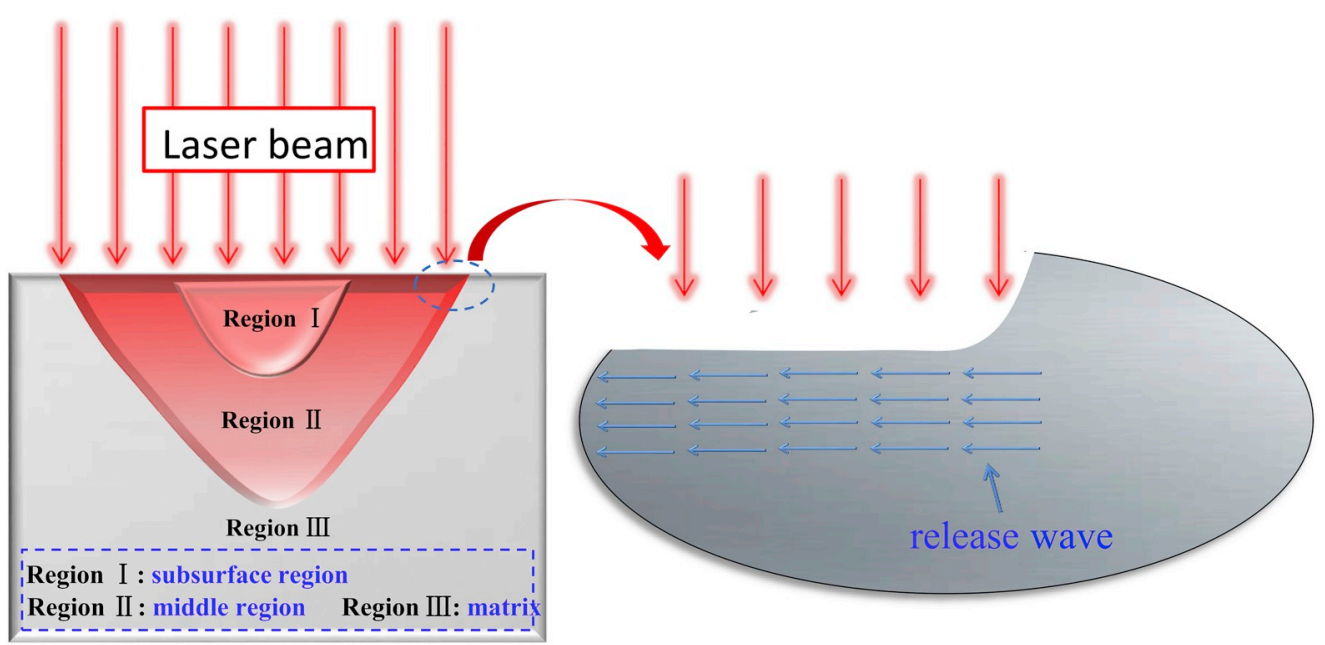

(b)
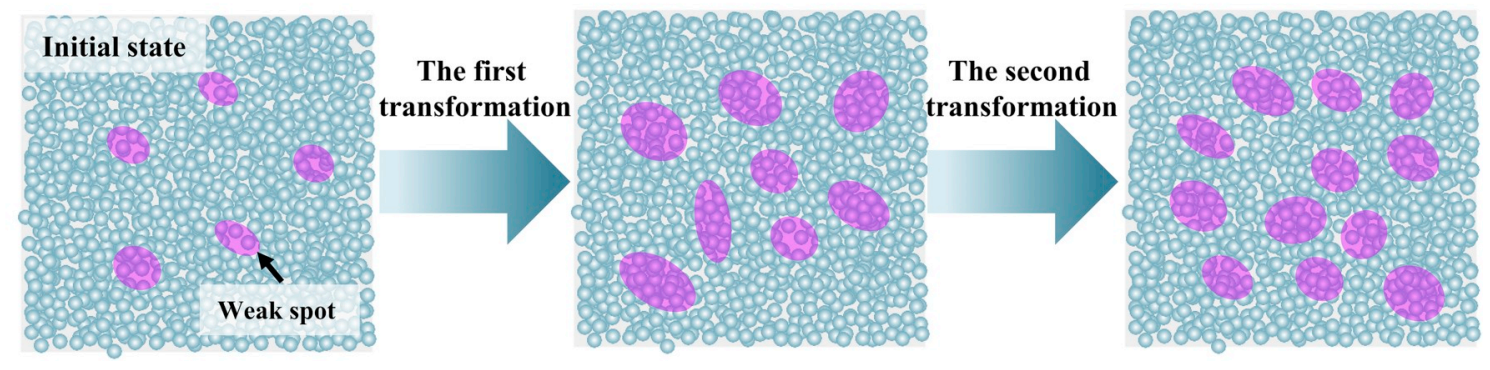

Fig. 5. Schematic illustrations of (a) the generation of the release wave by the boundary effect, (b) the two-step transformation process experienced by "weak spots" during NLSP process in the subsurface region. 
submitted.

\section{CRediT authorship contribution statement}

Yansen Li carried out the experimental work; Kun Zhang, Yansen Li and Bingchen Wei analyzed the data and wrote the manuscript; Yang Wang, Weiqi Tang, Yating Zhang and Zheng Hu provided experimental support; Bingchen Wei supervised the project overall and contributed to the discussion and direction of the research.

\section{Data availability}

The datasets generated during and/or analyzed during the current study are available from the corresponding author on reasonable request.

\section{Declaration of competing interest}

We declare that we do not have any commercial or associative interest that represents a conflict of interest in connection with the work submitted.

\section{Acknowledgements}

This work was supported by the National Natural Science Foundation of China (Grant No. 51401028, No.51271193, No. 11402277, No. 11790292), the Strategic Priority Research Program of the Chinese Academy of Sciences (Grant No. XDB22040303), and the Innovation Program (237099000000170004).

\section{References}

[1] A. Gujba, M. Medraj, Laser peening process and its impact on materials properties in comparison with shot peening and ultrasonic impact peening, Materials 7 (2014) 7925-7974.

[2] E. Williams, N. Lavery, Laser processing of bulk metallic glass: a review, J. Mater. Process. Technol. 247 (2017) 73-91.

[3] Y. Liao, C. Ye, G.J. Cheng, A review: warm laser shock peening and related laser processing technique, Opt. Laser. Technol. 78 (2016) 15-24.

[4] R. Fabbro, P. Peyre, L. Berthe, X. Scherpereel, Physics and applications of lasershock processing, J. Laser Appl. 10 (1998) 265-279.

[5] C.S. Montross, T. Wei, L. Ye, G. Clark, Y.W. Mai, Laser shock processing and its effects on microstructure and properties of metal alloys: a review, Int. J. Fatigue 24 (2002) 1021-1036.

[6] I. Altenberger, R.K. Nalla, Y.J. Sano, L. Wagner, R.O. Ritchie, On the effect of deeprolling and laser-peening on the stress-controlled low-and high-cycle fatigue behavior of Ti-6Al-4V at elevated temperatures up to $550 \mathrm{C}$, Int. J. Fatigue 44 (2012) 292-302.

[7] Y.W. Fang, Y.H. Li, W.F. He, P.Y. Li, Effects of laser shock processing with differen parameters and ways on residual stresses fields of a TC4 alloy blade, Mater. Sci. Eng. A 559 (2013) 683-692.

[8] X.F. Nie, W.F. He, L.C. Zhou, Q.P. Li, X.D. Wang, Experiment investigation of lase shock peening on TC6 titanium alloy to improve high cycle fatigue performance, Mater. Sci. Eng. A 594 (2014) 161-167.

[9] S.J. Lainé, K.M. Knowles, P.J. Doorbar, R.D. Cutts, D. Rugg, Microstructural characterisation of metallic shot peened and laser shock peened Ti-6Al-4V, Acta Mater. 123 (2017) 350-361.

[10] J. Fu, Y.H. Zhu, C. Zheng, R. Liu, Z. Ji, Effect of laser shock peening on the compressive deformation and plastic behavior of Zr-based bulk metallic glass, Opt. Lasers Eng. 86 (2016) 53-61.

[11] Y.H. Zhu, J. Fu, C. Zheng, Z. Ji, Structural and mechanical modifications induced on Zr-based bulk metallic glass by laser shock peening, Opt. Laser. Technol. 86 (2016) 54-60.

[12] Y.S. Li, Y.P. Wei, K. Zhang, Y.T. Zhang, Y. Wang, W.Q. Tang, Rejuvenation, embryonic shear bands and improved tensile plasticity of metallic glasses by nanosecond laser shock wave, J. Non-Cryst. Solids 513 (2019) 76-83.

[13] Y.P. Wei, G.Y. Xu, K. Zhang, Z. Yang, Y. Guo, C.G. Huang, B.C. Wei, Anomalous shear band characteristics and extra-deep shock-affected zone in Zr-based bulk metallic glass treated with nanosecond laser peening, Sci. Rep. 7 (2017), 43948.

[14] S. Zhao, B. Kad, B.A. Remington, J.C. LaSalvia, C.E. Wehrenberg, K.D. Behler, M. A. Meyers, Directional amorphization of boron carbide subjected to laser shock compression, Proc. Natl. Acad. Sci. 113 (2016) 12088-12093.
[15] A. Loveridge-Smith, A. Allen, J. Belak, T. Boehly, A. Hauer, B. Holian, D. Kalantar, G. Kyrala, R.W. Lee, P. Lomdahl, M.A. Meyers, D. Paisley, S. Pollaine, B. Remington, D.C. Swift, S. Weber, J.S. Wark, Anomalous elastic response of silicon to uniaxial shock compression on nanosecond time scales, Phys. Rev. Lett. 86 (2001) 2349.

[16] R. Sun, L. Li, Y. Zhu, L. Zhang, W. Guo, P. Peng, B. Li, C. Guo, L. Liu, Z.G. Che, Dynamic response and residual stress fields of Ti6Al4V alloy under shock wave induced by laser shock peening, Model Simul. Mater. Soc. 25 (2017), 065016.

[17] X.C. Zhanga, Y.K. Zhang, J.Z. Lu, F.Z. Xuan, Z.D. Wang, S.T. Tu, Improvement of fatigue life of Ti-6Al-4V alloy by laser shock peening, Mater. Sci. Eng. A 527 (2010) 3411-3415.

[18] Y.H. Hu, C. Gong, Z.Q. Yao, J. Hu, Investigation on the non-homogeneity of residual stress field induced by laser shock peening, Surf. Coat. Technol. 203 (2009) 3503-3508.

[19] A. Gujba, M. Medraj, Laser peening process and its impact on materials properties in comparison with shot peening and ultrasonic impact peening, Materials 7 (2014) 7925-7974.

[20] Y.M. Wang, E.M. Bringa, J.M. McNaney, M. Victoria, A. Caro, A.M. Hodge, R. Smith, B. Torralva, B.A. Remington, Deforming nanocrystalline nickel at ultrahigh strain rates, Appl. Phys. Lett. 88 (2006), 061917.

[21] M.A. Meyers, F. Gregori, B.K. Kad, M.S. Schneider, D.H. Kalantar, B.A. Remington, G. Ravichandran, T. Boehly, J.S. Wark, Laser-induced shock compression of monocrystalline copper: characterization and analysis, Acta Mater. 51 (2003) 1211-1228.

[22] H.B. Ke, J.F. Zeng, C.T. Liu, Y. Yang, Structure heterogeneity in metallic glass: modeling and experiment, J. Mater. Sci. Technol. 30 (2014) 560-565.

[23] L. Wang, L. Wang, Z. Nie, Y. Ren, Y.F. Xue, R.H. Zhu, H.F. Zhang, H.M. Fu, Evolution of residual stress, free volume, and hardness in the laser shock peened Tibased metallic glass, Mater. Des. 111 (2016) 473-481.

[24] Y. Cao, X. Xie, J. Antonaglia, B. Winiarski, G.Y. Wang, Y.C. Shin, P.J. Withers, K. A. Dahmen, P.K. Liaw, Laser shock peening on Zr-based bulk metallic glass and its effect on plasticity: experiment and modeling, Sci. Rep. 5 (2015), 10789.

[25] H. Huang, J. Zhang, C.H. Shek, J. Yan, Effects of pre-compression deformation on nanoindentation response of $\mathrm{Zr}_{65} \mathrm{Cu}_{15} \mathrm{Al}_{10} \mathrm{Ni}_{10}$ bulk metallic glass, J. Alloy. Comp. 674 (2016) 223-228.

[26] Y. Ma, J.H. Ye, G.J. Peng, D.H. Wen, T.H. Zhang, Nanoindentation study of size effect on shear transformation zone size in a Ni-Nb metallic glass, Mater. Sci. Eng. A 627 (2015) 153-160.

[27] Y.I. Golovin, Nanoindentation and mechanical properties of solids in submicrovolumes, thin near-surface layers, and films: a Review, Phys. Solid State 50 (2008) 2205-2236.

[28] B. Dubrujeaud, A. Fontes, P. Forget, C. Papaphilippou, C. Sainte-Catherine, M. Vardavoulias, M. Jeandin, Surface modification using high power lasers: influence of surface characteristics on properties of laser processed materials, Surf. Eng. 13 (1997) 461-470.

[29] Y.W. Bao, W. Wang, Y.C. Zhou, Investigation of the relationship between elastic modulus and hardness based on depth-sensing indentation measurements, Acta Mater. 52 (2004) 5397-5404.

[30] W.H. Wang, The elastic properties, elastic models and elastic perspectives of metallic glasses, Prog. Mater. Sci. 57 (2012) 487-656.

[31] A.L. Greer, Y.Q. Cheng, E. Ma, Shear bands in metallic glasses, Mater. Sci. Eng. R 74 (2013) 71-132.

[32] C.A. Schuh, T.G. Nieh, A nanoindentation study of serrated flow in bulk metallic glasses, Acta Mater. 51 (2003) 87-99.

[33] C.E. Packard, C.A. Schuh, Initiation of shear bands near a stress concentration in metallic glass, Acta Mater. 55 (2007) 5348-5358.

[34] W.L. Johnson, K. Samwer, A universal criterion for plastic yielding of metallic glasses with a (T/T g) 2/3 temperature dependence, Phys. Rev. Lett. 95 (2005), 195501.

[35] C.A. Schuh, A.C. Lund, Application of nucleation theory to the rate dependence of incipient plasticity during nanoindentation, J. Mater. Res. 19 (2004) 2152-2158.

[36] I.C. Choi, Y.K. Zhao, B.G. Yoo, Y.J. Kim, J.Y. Suh, U. Ramamurty, J. Jang, Estimation of the shear transformation zone size in a bulk metallic glass through statistical analysis of the first pop-in stresses during spherical nanoindentation, Scr. Mater. 66 (2012) 923-926.

[37] S.V. Ketov, Y.H. Sun, S. Nachum, Z. Lu, A. Checchi, A.R. Beraldin, H.Y. Bai, W. H. Wang, D.V. Louzguine-Luzgin, M.A. Carpenter, A.L. Greer, Rejuvenation of metallic glasses by non-affine thermal strain, Nature 524 (2015) 200.

[38] S. Couturier, T. de Rességuier, M. Hallouin, J.P. Romain, F. Bauer, Shock profile induced by short laser pulses, J. Appl. Phys. 79 (1996) 9338-9342.

[39] P. Peyre, R. Fabbro, Laser shock processing: a review of the physics and applications, Opt. Quant. Electron. 27 (1995) 1213-1229.

[40] V.V. Zhakhovskii, N.A. Inogamov, Elastic-plastic phenomena in ultrashort shock waves, JETP Lett. 92 (2010) 521-526.

[41] R. Fabbro, P. Peyre, L. Berthe, X. Scherpereel, Physics and applications of lasershock processing, J. Laser Appl. 10 (1998) 265-279.

[42] J. Antonaglia, X. Xie, G. Schwarz, M. Wraith, J. Qiao, Y. Zhang, P.K. Liaw, J.T. Uhl, K.A. Dahmen, Tuned critical avalanche scaling in bulk metallic glasses, Sci. Rep. 4 (2014) 4382. 